\title{
Comorbidity Between Psychiatric Diseases and Alcohol Use Disorders: Impact of Adolescent Alcohol Consumption
}

\author{
Jérôme Jeanblanc ${ }^{1,2}$
}

Published online: 28 September 2015

(C) Springer International Publishing AG 2015

\begin{abstract}
Patients suffering from psychiatric disorders such as schizophrenia and bipolar disorders often suffer from alcohol abuse or dependence. Conversely, most alcoholdependent patients suffer from other psychiatric disorders including depressive or anxiety disorders, with increased risk of suicide ideation and attempts. Adolescence is a crucial period of brain maturation but also of vulnerability to alcohol use disorders and other psychiatric diseases. While global alcohol consumption is decreasing in numerous Western countries, adolescent drinking is increasing with specific patterns of drinking called "binge drinking" leading to massive intoxication in a short period of time. Clinical and preclinical studies investigating the relationship between adolescent alcohol consumption and subsequent psychiatric disorders in patients or in animal models of psychiatric disorders will be described here and discussed.
\end{abstract}

Keywords Alcohol · Adolescence · Mental illness · Adulthood · Comorbidity

This article is part of the Topical Collection on Dual Diagnosis

Jérôme Jeanblanc

jerome.jeanblanc@u-picardie.fr

1 Research Group on Alcohol and Pharmacodependences - INSERM ERi24, UFR Pharmacie Université de Picardie Jules Verne, Amiens, France

2 Université de Picardie Jules Verne, Equipe INSERM ERI24, Groupe de Recherche sur l'Alcool et les Pharmacodépendances, GRAP, Centre de Recherche Universitaire en Santé (CURS), Chemin du Thil, 80025 Amiens Cedex 1, France

\section{Introduction}

Alcohol use disorders (AUDs), including alcohol abuse and alcohol dependence, are progressive mental disorders with high prevalence in the general population. The World Health Organization revealed that more than 76 million people worldwide have AUDs among the 2 billion consuming alcohol [1]. AUDs are responsible for 5 million deaths per year [2]. Although the prevalence of AUDs is variable across studies and countries, many populations show lifetime prevalence for AUDs of around $10 \%$. For example, the NCS-R showed a lifetime prevalence for alcohol abuse at around $13 \%$ and for alcohol dependence at about $5 \%$ [3]. Similarly, the National Epidemiologic Survey on Alcohol and Related Conditions (NESARC) reported a lifetime prevalence of around $18 \%$ for alcohol abuse and $12 \%$ for alcohol dependence [4].

AUDs also produce for a significant economic burden on society. The direct and indirect costs of AUDs reach more than $2 \%$ of gross domestic product in high- and middle-income countries [5]. Despite this impact, AUDs have the widest treatment gap among other psychiatric disorders with $78 \%$ of patients effected remaining untreated [6]. Furthermore, patients suffering from AUDs show high rates of relapse despite treatment: $38 \%$ of patients in treatment will relapse within 3 years after achieving abstinence [7].

Interestingly, another particularity of AUD patients is that $50-70 \%$ of them will suffer during their life from a comorbid psychiatric disorder [8], where the term "comorbidity" describes the simultaneous co-occurrence of two different behavioral health diagnoses in the same individual. Here, we will focus on the co-occurrence of any non-substance use psychiatric disorder related to alcohol dependence and vice versa. The relationship between these two disease entities has been understood in various ways that have differential implications for prevention and treatment: 
1. Both disorders are totally independent and can/should be treated separately

2. Psychiatric disorders are the consequences of alcohol use

3. AUD's are the consequence of psychiatric disorders:

(a) Because mentally ill people use ethanol (ETOH) to self-medicate

(b) Because mental illness worsens vulnerability to ETOH addiction

4. Both AUDs and mental illnesses share similar psychobiologic underlying mechanisms (e.g., they are actually one disease with two components)

In our perspective, the most probable explanations are, first, the self-medication hypothesis but with serious limitations and, second, the fact that AUDs might be another symptom of an existing psychiatric disorder. The self-medication hypothesis can explain for example the use of alcohol acutely to alleviate anxiety (thanks to its anxiolytic properties); however, chronic alcohol intake will have opposite effects and worsen the disease. In addition there is no correlation between the class of drug used and the symptoms that they are supposed to treat (psychostimulant during psychotic episode in schizophrenia, or use of heroin or cannabis during depressive episodes).

The prognosis for comorbid patients is more negative compared with that for non-comorbid patients. The co-occurrence of alcohol (or drug) dependence with other psychiatric disorders worsens psychiatric symptoms, increases their frequency, increases the number of days of hospitalization, and reduces life expectancy [9-12]. A better understanding of such comorbidity is therefore a pressing need that must be met to provide better care of patients and improved outcomes.

Adolescence is a window of vulnerability not only for onset of mental illness but also for the onset of risky behaviors and pursuit of new experiences including the use of alcohol and nicotine. The aim of this review is to compile evidence investigating a potential role for early alcohol exposure on the course of mental illnesses in alcohol dependent patients and in animal models of alcohol addiction.

\section{Comorbid Psychiatric Disorders in Alcohol-Dependent Patients}

\section{Depressive Disorders}

Depressive disorders are mood disorders characterized by sadness, despair, lack of desire to live, social withdrawal, loss of motivation, and, in severe cases, suicidal thinking and action. Depressive disorders are classified in the Diagnostic and Statistical Manual of Mental Disorders $\left(\mathrm{DSM}_{5}\right)$ as disruptive mood dysregulation disorder (for children older than 8 years old and up to 18), premenstrual dysphoric disorders, and persistent depressive disorder (the former dysthymia and major depressive disorders). Major depressive disorders occur in about $17 \%$ of the general population [13] but, in alcoholdependent patients, are more prevalent (e.g., ranging from $32 \%$ [8] to more than $42 \%$ [14]). As previously mentioned, the relationship between depression and AUDs may be quite complex and bidirectional. This relationship may also be differentially expressed or modulated by gender [8] and the poorer outcome observed might be due to their lower sensitivity to antidepressant treatment [15].

\section{Anxiety}

Anxiety is a normal reaction to stress that can often be adaptive in everyday life. However, in some people, anxiety becomes disproportionate and debilitating, producing loss of social, family, and professional functions. Anxiety disorders are among the most common types of mental disorders (or symptoms of mental disorders) including several wellcharacterized clinical entities such as panic disorder, social phobia, specific phobias, obsessive-compulsive disorder, and posttraumatic stress disorder. Anxiety disorders have a higher lifetime prevalence in women $(30.5 \%)$ as compared to men (19.2\%) [16]. In regards to the comorbidity of anxiety disorders with AUDs, a recent meta-analysis revealed a strong association between any anxiety disorder and both alcohol abuse (odds ratio of 1.636) and alcohol dependence (odds ratio of 2.532) [17]. Among the different types of anxiety disorders, social anxiety has been relatively well studied. Results obtained from the National Co-morbidity Study indicate lifetime prevalence of alcohol dependence about $24 \%$ in patients with social anxiety disorders (10\% more than patients without social anxiety disorders). Grant and colleagues found that $13 \%$ of patients suffering from social anxiety disorder within the last year, and more than $48 \%$ with lifetime social anxiety disorders met criterion for AUDs [18]. Globally, the different studies revealed that patients with social anxiety disorders have between two and three times more risk to develop AUDs in their life than persons without social anxiety disorders (see [19] for a review) and "coping" often being cited as the main motive to consume alcohol [20]. On the other hand, patients with AUDs multiply by 10 their risk to develop social anxiety disorders as compared to persons without AUDs [8]. Anxiety disorders can also appear in alcohol-dependent patients during specific periods of withdrawal $[21,22]$ in which patients cite that relapse provides relief from these anxious symptoms thanks to the anxiolytic properties of alcohol. Posttraumatic stress disorders (PTSDs) that may appear after lifethreatening events (war, car accident, sexual aggression) have also been extensively investigated in the past 15 years. Between 3 and $7 \%[3,23]$ of the general population will develop PTSD during their life. A recent systematic review of the 
literature indicates that among patients with PTSD, 10 to $61 \%$ suffer from comorbid alcohol misuse [24]. An imagery study demonstrated that AUDs enhance the structural alterations of the hippocampus induced by PTSD [25].

\section{Suicide}

Suicide rates vary between countries from around 3-4 per 100, 000 persons in South Africa to more than 30 per 100,000 persons in South Korea with a majority of countries having a suicidal rate around 10 to 15 per 100,000 persons [26]. This variability can be explained by cultural reasons (e.g., the stigma of the suicidal attempt for the family) or by criterion used to certify the cause of death. Suicide is three to four times more prevalent in men than in women, and suicide may be encountered at any age. However, two peaks of prevalence emerge: during adolescence and in elderly people ( $>60$ years old). Although suicide happens across demographic groups without "class distinction," suicide levels are highest among retirees, unemployed, divorced, urban people, and people living alone. A recent South Korean study revealed significant associations between Alcohol Use Disorders Identification Test (AUDIT) scores of 20 or over and suicidal ideation (odds ratio of 1.68) and suicidal attempts (odds ratio of 2.64) [27]. These authors also found that high frequency of drinking per week (more than four times) is significantly associated with suicide attempts (odds ratio of 2.85). Darvishi and colleagues [28] performed a meta-analysis of scientific publications focusing on the relationship between alcohol consumption and risk of suicide (ideation, attempt, and completed suicide). In this meta-analysis, the authors included a total of 31 studies with more than 400,000 subjects and found that AUDs were significantly associated with suicidal ideation (odds ratio of 1.86), suicide attempts (odds ratio of 3.13), and completed suicide (odds ratio of 2.59). The relationship between suicide and AUDs is complex since patients suffering from AUDs can secondarily develop major depressive disorders which can lead to suicide attempts, whereas patients with depression may sometimes claim that alcohol relieves their depressive symptoms.

\section{Bipolar Disorder}

Formerly called manic-depressive illness, bipolar disorder is part of the mood disorder spectrum. In its typical form, the patient experiences cycles between two different phases - the manic phase and the depressive phase - deviating from the normal (euthymic) state in various episodic patterns. During the manic phase, the patient is euphoric, hyperactive, and disinhibited and will suddenly have multiple projects to conduct in parallel. The depressive phase is characterized by sadness and lack of motivation and, in the most severe cases, similar to "pure" depressive disorders, leading to suicide ideation and/or attempts. Numerous studies found high rates of comorbid AUDs in patients suffering from bipolar disorders. The Epidemiological Catchment Area Study, conducted in the USA, found that bipolar patients have a $46 \%$ lifetime prevalence of AUDs [29]. The National Comorbidity Study, also conducted in the USA, revealed that patients with a diagnosis of alcohol dependence during their life have higher risk of developing comorbid mania [8]. More recently, Merikangas et al. [30] reported from more than 9000 patients included in the National Comorbidity Survey Replication a 39.1 and $23.2 \%$ lifetime prevalence of alcohol abuse and alcohol dependence, respectively. Interestingly, manic symptoms in general as well as bipolar type II disorders (mild manias, with intervening depressions) are strong predictors of lifetime alcohol abuse and alcohol dependence [31]. In Brazil, the prevalence rates of AUDs in patients diagnosed for bipolar disorders are similar to the previous studies and around $23 \%$ [32].

\section{Schizophrenia}

Schizophrenia is characterized by a series of symptoms classified as positive (hallucinations, delusion thought disorders), negative (social withdrawal, loss of motivation, neglect of personal hygiene), and cognitive symptoms (attention and memory deficits). About $1 \%$ of the total population suffers from schizophrenia, occurring in both men and women equally. Substance use disorders (SUDs) are well described in patients with schizophrenia, and studies show that almost all patients with schizophrenia have at least one addiction ( $85 \%$ to nicotine [33], 15 to $50 \%$ to cocaine [34], 12 to $42 \%$ to cannabis [34]. Concerning AUDs, the prevalence reaches more than $40 \%$ [35] to even $77 \%$ in one study [36]. However, a recent Norwegian study [37] based on a national registry covering all patients receiving public specialist health care in Norway (Norwegian Patients Register) found lower prevalence for schizophrenia $(0.34 \%)$ and for comorbid AUDs in patients with schizophrenia (4.6\%). The authors raised some limitations to their study including the fact that "the study only included people who had been in contact with specialist health care services during 5 years" and that "diagnoses of psychotic disorders and SUD in the NPR have not yet been subjected to formal validity testing." It is noteworthy that a large proportion of patients with schizophrenia already met criteria for AUDs according to the DSM-IV at the time of their first psychotic episode [38, 39] suggesting an early onset for AUDs in patients with schizophrenia.

\section{Alcohol Consumption in Adolescence}

Adolescence is a transition period between an infantile dependence and a more independent position. This period is marked by significant biological, neuro-anatomical, psychological, social, and behavioral changes [40]. The WHO defines an 
adolescent as a teenager aged between 10 and 19 years. Adolescence is triggered by the onset of puberty and theoretically ends after the consolidation of the final stages of physical development (17-21 years on average). However, periadolescent brain modifications continue until the age of 25 years old [41].

Global alcohol consumption has decreased over the two last decades in many countries (e.g., Italy, France, Japan, and other Southern European countries [42]). However, alcohol consumption among adolescents is increasing in almost all countries. The European Survey Health Behaviour in Schoolaged Children (HBSC) indicates that adolescents enrolled in middle school (11 to 16 years old mainly) have high levels of testing alcoholic drinks. Indeed, more than half of students in the sixth grade have already tasted an alcoholic drink (59.3\%) and $83.2 \%$ have by ninth grade [43]. Also, according to the results of this survey, $10 \%$ of male students and $6 \%$ of female students reported drinking alcohol at least once a week at the age of 13 . This proportion reaches $25 \%$ (for the males) and $13 \%$ (for the females) at the age of 15 . Thus, during middle school, the most frequently experienced psychoactive substance is alcohol, well ahead of tobacco and cannabis. Over the time spent of middle school, one out of six European students will experience at least one alcohol intoxication with levels multiplied by 5 between the sixth and the ninth grade ( 6.8 to $34 \%$ ) [43, 44]. A similar phenomenon is observed in the USA with the highest prevalence of alcohol dependence among 18 to 20 year olds [45]. The National Survey on Drug Use and Health indicates that from age of 12 to age of 21, the prevalence of alcohol use increases from about $10 \%$ to more than $85 \%$ [46]. This makes alcohol, similar to the European context, the drug also most used by American teenagers, way above tobacco and marijuana [47].

All these data indicate that adolescents consume high levels of alcohol. But, in addition to this, the pattern of drinking itself is different from that of adult consumers. Indeed adolescents drink less frequently but more heavily at each episode of consumption. This phenomenon, called binge drinking, is characterized by ingesting a large amount of alcohol in a short period of time, to rapidly get drunk. Particularly present in adolescents and young adults, this mode of consumption is precisely defined by the National Institute on Alcohol Abuse and Alcoholism [48] as consumption within $2 \mathrm{~h}$ of a large amount of alcohol corresponding to $60 \mathrm{~g}$ of pure alcohol (six glasses) for women and $70 \mathrm{~g}$ (seven glasses) for men which leads to blood alcohol levels greater or equal to $0.8 \mathrm{~g} / \mathrm{l}$. This mode of consumption leading to repeated drunkenness is particularly harmful at both cognitive $[49,50]$ and neuroanatomical levels (for a review, see [51]).

The particular vulnerability of adolescents toward alcohol can be explained by the late maturation of specific brain regions such as the prefrontal cortex involved in inhibitory control of actions and in evaluation of action consequences. This delayed maturation of frontal brain regions has been shown to be associated with activation of the amygdala involved in emotions, conditioning, and fear [52]. Normal adolescent development reverses this balance to restore the highest activity of the frontal cortical regions with a decrease in the amygdala [53]. However, early alcohol consumption may disrupt this process of maturation as it has been demonstrated that adolescents who misuse alcohol show poor performance in inhibition tasks and mental flexibility [54]. Another study compared the neuropsychological performance of four different groups of subjects: young binge drinkers, young moderate drinkers, abstinent young adults, and healthy elderly subjects (mean age of 69 years old). The authors found that binge drinkers have poorer performance than moderate drinkers or abstinent subjects, but comparable performances to older people [49].

\section{Late Psychiatric Consequences of Adolescent Alcohol Consumption}

Except for subsequent alcohol dependence induced by an early alcohol initiation (which is not the scope of this review), very few studies have investigated the impact of adolescent exposure to alcohol on other adult psychiatric disorders commonly found to be comorbid with AUDs.

Brook and colleagues [55] found in a longitudinal study that alcohol consumption during adolescence increases the odds for adult diagnosis of anxiety disorders (odds ratio of 1.30) and antisocial personality disorders (odds ratio of 1.36). McCambridge and colleagues performed a systematic review of studies investigating the late consequences of alcohol use during adolescence. Thirty-five reports were generated from 10 different cohorts and 19 other reports from individual cohorts [56]. From these 54 reports, only 5 investigated the association between early alcohol consumption and late consequences on psychiatric disorders other than AUDs. These five studies specifically focused on depressive disorders, anxiety disorders, and suicidal ideation/attempts. Taken together, most of the studies demonstrated a strong association between alcohol consumption during adolescence with adult (or young adult) diagnoses of depression or anxiety disorders [57-60]. However, two studies did not find a link between early/ adolescent alcohol consumption and depressive disorders or anxiety disorders later on $[59,61]$. Rohde et al. found that adolescents with alcohol problems (defined as "one or two symptoms of alcohol dependence but no AUD diagnosis" established [58]) exhibit higher risk for antisocial personality disorders. In regards with suicidal ideation or attempts, alcohol does not seem to have any effects at adulthood (age between 21 and 25) in distinction to its effect during late adolescence (age between 16 and 21) [59]. Thus, very few human studies have investigated the role of adolescent alcohol consumption on late psychiatric disorders other than alcohol 
abuse and dependence. Moreover, the "late" consequences are often evaluated in young adults, only a few years after the end of adolescence and rarely after 35-40 years old. Furthermore, the question of whether certain severe psychiatric disorders such as schizophrenia and bipolar disorders may be contributed to by heavy adolescent drinking has been almost completely unexplored in human studies.

\section{What do Animal Models Teach Us About the Impact of Alcohol Exposure During Adolescence?}

Obviously, some human psychiatric conditions described in this review cannot be studied using animal models, such as suicide ideation/attempts for example. Nonetheless, numerous animal models exist for psychiatric diseases (for a review, see [62]) which can be highly informative to clinical context when their strengths and weaknesses are carefully considered [63]. However, similar to human studies, only a few animal models of psychiatric disorders have been the subject of investigation aiming at identifying the impact of adolescence exposure to alcohol on subsequent occurrence of psychiatric symptoms or syndromes.

\section{Depression}

Over the last 5 years, insufficient data has been published to make any conclusions on the potential role for alcohol exposure during adolescence on adult depression-like behavior. However, Ribiero-Carvalhi and his colleagues [64] used a protocol of intra-peritoneal injections of alcohol $(2 \mathrm{~g} / \mathrm{kg}$, $25 \%$ ethanol solution) every other day between the postnatal days 30 and 45 corresponding to adolescence in mice [65]. Depression-like behaviors were assessed with the Forced Swim Test and with a sucrose preference test. At adulthood, only a weak impact of early alcohol exposure in the forced Swim test was found in female mice, and no effect at all in both male and female mice in the sucrose preference test. The authors used the C57BL/6 strain of mice which is known to be an alcohol-preferring strain meaning that the mice spontaneously consume high levels of alcohol [66], and this specific phenotype toward alcohol might have interfered with specific onset on depression-like behavior. In addition, C57BL/6 mice exhibit already high level of immobility in the Forced Swim Test [67] as compared to other strains of mice inducing a potential ceiling effect.

\section{Anxiety}

As in humans, the relationship between alcohol consumption and anxiety has been more widely studied in rodents, but interestingly with diverse findings. Rats selectively bred for their alcohol-preferring phenotype can show either high [68] or low anxiety levels [69]. Conversely, two selected strains of rats for high levels of anxiety did not show increased levels of alcohol consumption [70]. As mentioned for humans, anxiety can be induced by alcohol withdrawal, and Zhang and colleagues have demonstrated in adult rats that acute and repeated alcohol administration induces anxiety-like behavior [71].

Recent studies have demonstrated a link between adolescent alcohol administration and subsequent anxiety-like behavior in adulthood. Using a binge drinking exposure paradigm during adolescence (postnatal days 28 to 53), MeijaToiber and colleagues [72] studied anxiety-like behavior at adulthood (postnatal days 185) in the light-potentiated startle test. It is noteworthy that rats were re-exposed to alcohol following the same 4-day binge drinking procedure just before the anxiety test. The authors found an elevated anxiety level during the withdrawal period in adult rats pre-exposed to alcohol during adolescence. Crews' group used intra-gastric administration of alcohol (5 g/kg, $20 \%$ ethanol solution) between postnatal days 25 and 55 on a 2-day on/2-day off schedule similar to the one developed by Pascual et al. [73] with intra-peritoneal injections. Alcohol-treated rats exhibited in advanced adulthood (postnatal day 219) higher anxiety measured as the time spent out of the light during a light/dark box test [74]. In another study, Pandey and his colleagues also used the Pascual et al. [73] protocol of intra-peritoneal injections between the age of 28 and 41 days on a 2-day on/2-day off schedule. Alcohol-treated rats displayed at the age of 92 days higher anxiety in the light/dark box and in the elevated plus maze tests. This high level of anxiety was already present during adolescence; thus, these results suggest that alcohol-induced anxiety observed in adolescents persists until adulthood and probably through modification of gene expression thanks to epigenetic mechanisms $[75 \bullet \bullet]$.

\section{Schizophrenia}

A large number of animal models of schizophrenia are available (genetic, pharmacological models, etc.), but in regards to studying comorbid use of substances in mental illness, the neonatal ventral hippocampal lesion (NVHL) model described by Lipska and Weinberger [76] has been the most frequently used. Chambers' group has largely investigated the adult consumption of drugs of abuse such as cocaine $[77,78]$, nicotine $[79,80]$, and also alcohol $[81,82]$ with this model showing increased consumption of the different drugs of abuse at adulthood. However, our group was the first to investigate the impact of adolescent alcohol exposure on subsequent AUDs in adulthood in the NVHL model of schizophrenia [83]. In this study, we found that moderate alcohol consumption using a $10 \%$ continuous access two-bottle choice paradigm during adolescence (postnatal days 28 to 42 ) is necessary to observe at adulthood a loss of control over alcohol consumption. This loss of control, found using a $20 \%$ 
intermittent access protocol, is associated with higher motivation to consume alcohol, resistance to extinction, and higher relapse after protracted abstinence in an operant selfadministration paradigm ( $20 \%$ ethanol solution). These specific phenotypes were long lasting since after more than a month, pre-exposed NVHL rats still exhibited higher levels of ethanol consumption $(0.9 \mathrm{~g} / \mathrm{kg} / 30 \mathrm{~min}$ vs. $0.4 \mathrm{~g} / \mathrm{kg} / 30 \mathrm{~min}$ for the pre-exposed SHAM rats). None of the behavioral alterations were observed either in control animals pre-exposed to alcohol, or in alcohol-pre-exposed NVHL rats tested in adulthood in terms of sucrose consumption. Further investigations are ongoing in order to assess the impact of these addiction-like phenotypes on schizophrenia-like behaviors.

\section{Decision Making}

Decision making is the ability to select the most favorable option for the subject among many at a particular time. This selection is based on several internal and external parameters. Decision making is crucial in everyday life to adapt our behavior to the changing environment. Abnormal decision making may be a key concept to understand behavioral disturbances present in depression [84], anxiety disorders [85], schizophrenia [86, 87], and other mental illnesses [88].

Preclinical studies have demonstrated that adolescent alcohol consumption induces alterations in decision making processes at adulthood. Nasrallah and his colleagues [89] preexposed adolescent rats (postnatal days 30 to 49 ) to alcohol (10\% ethanol geletin) and evaluated the risk-based decision making processes at adulthood ( 3 months after the end of alcohol exposure) using a probabilistic discounting task. Preexposed rats showed significantly higher risky choices than control rats associated with higher dopamine release in the core part of the nucleus accumbens. Moreover, the authors showed altered phasic dopamine release during the choices in pre-exposed rats. On the opposite, no difference of dopamine release was observed when rewards (sucrose pellets) were delivered freely to the animals. These results suggest that alcohol pre-exposure during adolescence modifies decision making in adulthood by altering dopamine-related encoding of the cost during the choice but not the consumption value of the reward. A study from the same group identified a dysregulation of learning processes involved in these decision making alterations induced by early alcohol consumption [90]. Indeed, pre-exposed rats showed fast learning for what the authors called "better-than-expected" rewards but not "worse-than-expected" ones still without altering the perception of the real value of the rewards. Using another alcohol pre-exposure procedure $(5 \mathrm{~g} / \mathrm{kg}$ of $25 \%$ ethanol intragastrically in a 2-day on/2-day off pattern), Boutros et al. found similar increased risky choice at adulthood and, within the prelimbic area of the prefrontal cortex, reduced levels of tyrosine hydroxylase, the enzyme responsible for the synthesis of dopamine. Likewise, the authors found reduced levels of choline acetyltransferase in cholinergic nuclei [91•].

\section{Conclusion}

AUDs in patients suffering from psychiatric disorders show increased severity and frequency of psychiatric symptoms complicating their care. Numerous studies have demonstrated that adolescent alcohol consumption is strongly associated with higher risk of premature death, and to acquiring AUDs during adulthood. Nonetheless, unlike cannabis use and schizophrenia, alcohol consumption during adolescence does not seem to influence the age-of-onset of major psychiatric disorders exhibited by alcohol-dependent patients. However, despite the scarcity of clinical and preclinical studies investigating the role of early alcohol consumption on adult frequency and severity of psychiatric disorders, it seems clear that alcohol consumption during adolescence is strongly associated to anxiety and depressive disorders at adulthood.

Considering the dramatic brain damages induced by early alcohol consumption observed in clinical and preclinical studies, it is perhaps surprising that a link between adult psychiatric diseases and early adolescent alcohol exposure has not been robustly observed. But, this lack of strong evidence might be due to the small number of studies investigating such a link. McCambridge et al. [56] reviewed 54 reports with only 5 focusing on psychiatric disorders other than alcohol dependence. Moreover, most of these studies focused on only mood disorders. Thus, we can imagine that with an increased number of studies, with specific focus on each psychiatric disorder or endophenotypes of each disease on a long lifespan, we may yet still detect long-lasting behavioral and psychiatric effects of adolescent alcohol consumption.

The small number of preclinical studies has already revealed very interesting information about the behavioral and neurochemical basis underlying the link between AUDs and psychiatric disorders. This suggests the need for greater efforts in examining the development of comorbid AUDs in already existing animal models of psychiatric disorders, despite the added technical complexity that this entails. Employment of cutting-edge techniques such as in vivo fast cyclic voltammetry, optogenetics, or Designer Receptors Exclusively Activated by Designer Drugs (DREADD) techniques to these preclinical models will allow new research and new scientific questions focusing on neurobiological basis of the comorbid use of alcohol in animal models of mental illness.

Nevertheless, in addition to the already demonstrated link between alcohol consumption during adolescence and mood disorders, early alcohol exposure leads to high risk of AUDs. Since AUDs in patients suffering from psychiatric diseases are associated with worse prognosis and poor treatment outcome, prevention against alcohol consumption in adolescents in 
general but specifically in populations at risk for any of the major psychiatric diseases may be important to improve patients' care at adulthood.

Comorbid AUDs in patients with mental illness is, sadly, still not enough evaluated as a clinical entity despite its significant prevalence. In most treatment facilities, patients can only be admitted for a psychiatric disorder in a psychiatric facility, or in an addiction treatment center for their addiction issues. Only rarely are patients treated for both diseases at the same time and at the same place. In addition, an effort in training more medical staff to take care of such comorbid patients is necessary to achieve efficient care of this particular population of patients. Pursuing clinical and preclinical research on AUDs comorbid with psychiatric disorders, and achieving these efforts in the context of integrated organization and training structures will be needed to improve the prognosis of patients suffering from both substance use disorders and mental illnesses.

\section{Compliance with Ethics Guidelines}

Conflict of Interest Jérôme Jeanblanc declares no conflict of interest.

Human and Animal Rights and Informed Consent This article does not contain any studies with human subjects performed by any of the authors. Animal studies mentioned in this article performed by the authors were performed accordingly to the European community regulations for animal use in research (CEE No 86/609) and were approved by the local ethical committee (Comité Régional d'Ethique en Matière d'Expérimentation Animale de Picardie).

\section{References}

Papers of particular interest, published recently, have been highlighted as:

- Of importance

-• Of major importance

1. WHO Global Status Report on Alcohol 2004. World Health Organization Department of Mental Health and Substance Abuse Geneva.

2. Whiteford HA, Degenhardt L, Rehm J, Baxter AJ, Ferrari AJ, Erskine HE, et al. Global burden of disease attributable to mental and substance use disorders: findings from the Global Burden of Disease Study 2010. Lancet. 2013;382:1575-86.

3. Kessler RC, Berglund P, Demler O, Jin R, Merikangas KR, Walters EE. Lifetime prevalence and age-of-onset distributions of DSM-IV disorders in the National Comorbidity Survey Replication. Arch Gen Psychiatry. 2005;62:593-602.

4. Hasin DS, Stinson FS, Ogburn E, Grant BF. Prevalence, correlates, disability, and comorbidity of DSM-IV alcohol abuse and dependence in the United States: results from the National Epidemiologic
Survey on Alcohol and Related Conditions. Arch Gen Psychiatry. 2007;64:830-42.

5. Rehm J, Mathers C, Popova S, Thavorncharoensap M, Teerawattananon Y, Patra J. Global burden of disease and injury and economic cost attributable to alcohol use and alcohol-use disorders. Lancet. 2009;373:2223-33.

6. Kohn R, Saxena S, Levav I, Saraceno B. The treatment gap in mental health care. Bull World Health Organ. 2004;82:858-66.

7. Moos RH, Moos BS. Rates and predictors of relapse after natural and treated remission from alcohol use disorders. Addiction. 2006;101:212-22.

8. Kessler RC, Crum RM, Warner LA, Nelson CB, Schulenberg J, Anthony JC. Lifetime co-occurrence of DSM-III-R alcohol abuse and dependence with other psychiatric disorders in the National Comorbidity Survey. Arch Gen Psychiatry. 1997;54:313-21.

9. Margolese HC, Malchy L, Negrete JC, Tempier R, Gill K. Drug and alcohol use among patients with schizophrenia and related psychoses: levels and consequences. Schizophr Res. 2004;67:157-66.

10. Thoma P, Daum I. Comorbid substance use disorder in schizophrenia: a selective overview of neurobiological and cognitive underpinnings. Psychiatry Clin Neurosci. 2013;67:367-83.

11. Farren CK, Hill KP, Weiss RD. Bipolar disorder and alcohol use disorder: a review. Curr Psychiatry Rep. 2012;14:659-66.

12. Farren CK, Murphy P, McElroy S. A 5-year follow-up of depressed and bipolar patients with alcohol use disorder in an Irish population. Alcohol Clin Exp Res. 2014;38:1049-58.

13. Kessler RC, Chiu WT, Demler O, Merikangas KR, Walters EE. Prevalence, severity, and comorbidity of 12-month DSM-IV disorders in the National Comorbidity Survey Replication. Arch Gen Psychiatry. 2005;62:617-27.

14. Schuckit MA, Tipp JE, Bucholz KK, Nurnberger Jr JI, Hesselbrock VM, Crowe RR, et al. The life-time rates of three major mood disorders and four major anxiety disorders in alcoholics and controls. Addiction. 1997;92:1289-304.

15. Hashimoto E, Tayama M, Ishikawa $H$, Yamamoto $M$, Saito $T$. Influence of comorbid alcohol use disorder on treatment response of depressive patients. J Neural Transm. 2015;122:301-6.

16. Kessler RC, McGonagle KA, Zhao S, Nelson CB, Hughes M, Eshleman S, et al. Lifetime and 12-month prevalence of DSM-III$\mathrm{R}$ psychiatric disorders in the United States. Results from the National Comorbidity Survey. Arch Gen Psychiatry. 1994;51:8-19.

17. Lai HM, Cleary M, Sitharthan T, Hunt GE Prevalence of comorbid substance use, anxiety and mood disorders in epidemiological surveys, 1990-2014: A systematic review and meta-analysis. Drug Alcohol Depend. 2015

18. Grant BF, Hasin DS, Blanco C, Stinson FS, Chou SP, Goldstein $\mathrm{RB}$, et al. The epidemiology of social anxiety disorder in the United States: results from the National Epidemiologic Survey on Alcohol and Related Conditions. J Clin Psychiatry. 2005;66:1351-61.

19. Morris EP, Stewart SH, Ham LS. The relationship between social anxiety disorder and alcohol use disorders: a critical review. Clin Psychol Rev. 2005;25:734-60.

20. Ham LS, Bonin M, Hope DA. The role of drinking motives in social anxiety and alcohol use. J Anxiety Disord. 2007;21:9911003.

21. Allan CA. Alcohol problems and anxiety disorders-a critical review. Alcohol Alcohol. 1995;30:145-51.

22. Kushner MG, Abrams K, Borchardt C. The relationship between anxiety disorders and alcohol use disorders: a review of major perspectives and findings. Clin Psychol Rev. 2000;20:149-71.

23. McManus S, Meltzer H, Brugha T, Bebbington P, Jenkins R, editors. Adult psychiatric morbidity in England, 2007: results of a household survey. Leeds: The NHS Information Centre for Health and Social Care; 2009.

24. Debell F, Fear NT, Head M, Batt-Rawden S, Greenberg N, Wessely $\mathrm{S}$, et al. A systematic review of the comorbidity between PTSD and 
alcohol misuse. Soc Psychiatry Psychiatr Epidemiol. 2014;49(9): 1401-25.

25. Starčević A, Dimitrijević I, Aksić M, Stijak L, Radonjić V, Aleksić $\mathrm{D}$, et al. Brain changes in patients with posttraumatic stress disorder and associated alcoholism: MRI based study. Psychiatr Danub. 2015;27(1):78-83

26. OECD. "Suicides", in OECD Factbook 2013: Economic, Environmental and Social Statistics, OECD Publishing; 2013. 10. 1787/factbook-2013-97-en.

27. Bae HC, Hong S, Jang SI, Lee KS, Park EC. Patterns of Alcohol Consumption and Suicidal Behavior: Findings From the Fourth and Fifth Korea National Health and Nutritional Examination Survey (2007-2011). J Prev Med Public Health. 2015;48:142-50.

28. Darvishi N, Farhadi M, Haghtalab T, Poorolajal J. Alcohol-related risk of suicidal ideation, suicide attempt, and completed suicide: a meta-analysis. PLoS One. 2015;10:e126870.

29. Regier DA, Farmer ME, Rae DS, Locke BZ, Keith SJ, Judd LL, et al. Comorbidity of mental disorders with alcohol and other drug abuse. Results from the Epidemiologic Catchment Area (ECA) Study. JAMA. 1990;264:2511-8.

30. Merikangas KR, Akiskal HS, Angst J, Greenberg PE, Hirschfeld RM, Petukhova M, et al. Lifetime and 12-month prevalence of bipolar spectrum disorder in the National Comorbidity Survey replication. Arch Gen Psychiatry. 2007;64:543-52.

31. Merikangas KR, Herrell R, Swendsen J, Rossler W, Ajdacic-Gross $\mathrm{V}$, Angst J. Specificity of bipolar spectrum conditions in the comorbidity of mood and substance use disorders: results from the Zurich cohort study. Arch Gen Psychiatry. 2008;65:47-52.

32. Nery FG, Miranda-Scippa A, Nery-Fernandes F, Kapczinski F, Lafer B. Prevalence and clinical correlates of alcohol use disorders among bipolar disorder patients: results from the Brazilian Bipolar Research Network. Compr Psychiatry. 2014;55:1116-21.

33. Morisano D, Bacher I, Audrain-McGovern J, George TP. Mechanisms underlying the comorbidity of tobacco use in mental health and addictive disorders. Can J Psychiatry. 2009;54:356-67.

34. Chambers RA, Krystal JH, Self DW. A neurobiological basis for substance abuse comorbidity in schizophrenia. Biol Psychiatry. 2001;50:71-83.

35. Mueser KT, Yarnold PR, Bellack AS. Diagnostic and demographic correlates of substance abuse in schizophrenia and major affective disorder. Acta Psychiatr Scand. 1992;85:48-55.

36. Schmidt LM, Hesse M, Lykke J. The impact of substance use disorders on the course of schizophrenia-a 15-year follow-up study: dual diagnosis over 15 years. Schizophr Res. 2011;130:228-33.

37. Nesvag R, Knudsen GP, Bakken IJ, Hoye A, Ystrom E, Suren P, Reneflot A, Stoltenberg C, Reichborn-Kjennerud T. Substance use disorders in schizophrenia, bipolar disorder, and depressive illness: a registry-based study. Soc Psychiatry Psychiatr Epidemiol. 2015

38. Cantwell R, Brewin J, Glazebrook C, Dalkin T, Fox R, Medley I, et al. Prevalence of substance misuse in first-episode psychosis. Br J Psychiatry. 1999;174:150-3.

39. Buhler B, Hambrecht M, Loffler W, an der Heiden W, Hafner H. Precipitation and determination of the onset and course of schizophrenia by substance abuse-a retrospective and prospective study of 232 population-based first illness episodes. Schizophr Res. 2002;54:243-51.

40. Spear LP. Adolescents and alcohol: acute sensitivities, enhanced intake, and later consequences. Neurotoxicol Teratol. 2014;41:51-9.

41. Toga AW, Thompson PM, Sowell ER. Mapping brain maturation. Trends Neurosci. 2006;29:148-59.

42. OECD. "Alcohol consumption", in OECD Factbook 2013: Economic, Environmental and Social Statistics, OECD Publishing; 2013. 10.1787/factbook-2013-99-en.

43. Spilka S, Le Nézet O, Beck F, Ehlinger V, Godeau E. Alcool, tabac et cannabis durant les "années collège". Tendances. 2012; 80.
44. Currie C, Zanotti C, Morgan A, Currie D, De Looze M, Roberts C, et al. Social Determinants of Health and Well-Being Among Young People. Health Behaviour in School-Aged Children (HBSC) Study: International Report from the 2009/2010 Survey, vol. 6 . Copenhagen: WHO Regional Office for Europe; 2012.

45. Grant BF, Dawson DA, Stinston FS, Dufour MC, Pickering RP. The 12-month prevalence and trends in DSM-IV alcohol abuse and dependence: United States, 1991-1992 and 2001-2002. Drug Alcohol Depend. 2004;74:223-34.

46. Johnston LD, O'Malley PM, Bachman JG, et al. Monitoring the Future, National Survey Results on Drug Use, 1975-2005. Volume 1: Secondary School Students. NIH Pub. No. 06-5883. Bethesda: National Institute on Drug Abuse; 2006.

47. The Surgeon General's Call to Action To Prevent and Reduce Underage Drinking Office of the Surgeon General (US); National Institute on Alcohol Abuse and Alcoholism (US); Substance Abuse and Mental Health Services Administration (US). Rockville (MD): Office of the Surgeon General (US); 2007.

48. NIAAA, 2005 http://pubs.niaaa.nih.gov/publications/Newsletter/ winter2004/Newsletter Number3.htm\#council

49. Sanhueza C, Garcia-Moreno LM, Exposito J. Weekend alcoholism in youth and neurocognitive aging. Psicothema. 2011;23:209-14.

50. Peeters M, Vollebergh WA, Wiers RW, Field M. Psychological changes and cognitive impairments in adolescent heavy drinkers. Alcohol Alcohol. 2014;49:182-6.

51. Petit G, Maurage P, Kornreich C, Verbanck P, Campanella S. Binge drinking in adolescents: a review of neurophysiological and neuroimaging research. Alcohol Alcohol. 2014;49:198-206.

52. Gierski F, Naassila M. Giersky and Naassila 2015 Conduites d'alcoolisation et binge drinking chez l'adolescents : apport des neurosciences. In: Battaglia N, Gierski F, editors. L'addiction chez les adolescents: psychologie des conduites addictives. Paris: De Boeck - Solal; 2014

53. Ernst M, Pine DS, Hardin M. Triadic model of the neurobiology of motivated behavior in adolescence. Psychol Med. 2006;36:299-312.

54. Winward JL, Hanson KL, Tapert SF, Brown SA. Heavy alcohol use, marijuana use, and concomitant use by adolescents are associated with unique and shared cognitive decrements. J Int Neuropsychol Soc. 2014;20:784-95.

55. Brook JS, Cohen P, Brook DW. Longitudinal study of co-occurring psychiatric disorders and substance use. J Am Acad Child Adolesc Psychiatry. 1998:37:322-30.

56. McCambridge J, McAlaney J, Rowe R. Adult consequences of late adolescent alcohol consumption: a systematic review of cohort studies. PLoS Med. 2011;8:e1000413.

57. Andreasson S, Allebeck P. Alcohol and psychiatric illness: longitudinal study of psychiatric admissions in a cohort of Swedish conscripts. Subst Use Misuse. 1991;26:713-28.

58. Rohde P, Lewinsohn PM, Kahler CW, Seeley JR, Brown RA. Natural course of alcohol use disorders from adolescence to young adulthood. J Am Acad Child Adolesc Psychiatry. 2001;40:83-90.

59. Wells JE, Horwood LJ, Fergusso DM. Drinking patterns in midadolescence and psychosocial outcomes in late adolescence and early adulthood. Addiction. 2004;99:1529-41.

60. Mason W, Kosterman R, Haggerty KP, Hawkins J, Redmond C, et al. Dimensions of adolescent alcohol involvement as predictors of young-adult major depression. J Stud Alcohol Drugs. 2008;69: 275-85.

61. Kandel DB, Davies M, Karus D, Yamaguchi K. The consequences in young adulthood of adolescent drug involvement. An overview. Arch Gen Psychiatry. 1986;43(8):746-54.

62. Nestler EJ, Hyman SE. Animal models of neuropsychiatric disorders. Nat Neurosci. 2010;13:1161-9.

63. Wong AH, Josselyn SA. Caution When Diagnosing Your Mouse with Schizophrenia: The Use and Misuse of Model Animals for Understanding Psychiatric Disorders. Biol Psychiatry. 2015 
64. Ribeiro-Carvalho A, Lima CS, Nunes-Freitas AL, Filgueiras CC, Manhaes AC, Abreu-Villaca Y. Exposure to nicotine and ethanol in adolescent mice: effects on depressive-like behavior during exposure and withdrawal. Behav Brain Res. 2011;221:282-9.

65. Spear LP. Modeling adolescent development and alcohol use in animals. Alcohol Res Health. 2000;24:115-23.

66. Rhodes JS, Ford MM, Yu CH, Brown LL, Finn DA, Garland Jr T, et al. Mouse inbred strain differences in ethanol drinking to intoxication. Genes Brain Behav. 2007;6:1-18.

67. Can A, Dao DT, Arad M, Terrillion CE, Piantadosi SC, Gould TD. The mouse forced swim test. J Vis Exp. 2012;e3638.

68. Colombo G, Lobina C, Carai MA, Gessa GL. Phenotypic characterization of genetically selected Sardinian alcohol-preferring (sP) and -non-preferring (sNP) rats. Addict Biol. 2006;11:324-38.

69. Acewicz A, Mierzejewski P, Jastrzebska A, Korkosz I, Karas K, Sienkiewicz-Jarosz H, et al. Anxiety- and depressive-like traits in Warsaw alcohol high-preferring (WHP) and Warsaw alcohol lowpreferring (WLP) rats. Pharmacol Biochem Behav. 2014;122:261-5.

70. Da Silva GE, Ramos A, Takahashi RN. Comparison of voluntary ethanol intake by two pairs of rat lines used as genetic models of anxiety. Braz J Med Biol Res. 2004;37:1511-7.

71. Zhang Z, Morse AC, Koob GF, Schulteis G. Dose- and timedependent expression of anxiety-like behavior in the elevated plusmaze during withdrawal from acute and repeated intermittent ethanol intoxication in rats. Alcohol Clin Exp Res. 2007;31:1811-9.

72. Mejia-Toiber J, Boutros N, Markou A, Semenova S. Impulsive choice and anxiety-like behavior in adult rats exposed to chronic intermittent ethanol during adolescence and adulthood. Behav Brain Res. 2014;266:19-28.

73. Pascual M, Boix J, Felipo V, Guerri C. Repeated alcohol administration during adolescence causes changes in the mesolimbic dopaminergic and glutamatergic systems and promotes alcohol intake in the adult rat. J Neurochem. 2009;108:920-31.

74. Vetreno RP, Yaxley R, Paniagua B, Crews FT. Diffusion tensor imaging reveals adolescent binge ethanol-induced brain structural integrity alterations in adult rats that correlate with behavioral dysfunction. Addict Biol. 2015. doi:10.3389/fnins.2015.00035.

75.• Pandey SC, Sakharkar AJ, Tang L, Zhang H. Potential role of adolescent alcohol exposure-induced amygdaloid histone modifications in anxiety and alcohol intake during adulthood. Neurobiol Dis. 2015. doi:10.1016/j.nbd.2015.03.019. The authors demonstrated a long lasting effect of adolescent exposure to alcohol and identified potential molecular modifications responsible for these effects involving epigenetic mechanisms. Authors found decreased BDNF exons I and IV promoter histone $\mathrm{H3}$ acetylation associated to decreased BDNF expression within the amygdala at adulthood after alcohol exposure. Finally, they decreased anxiety-like behaviors and restored $\mathrm{H} 3$ acetylation of BDNF promoters with trichostatin treatment

76. Lipska BK, Weinberger DR. Delayed effects of neonatal hippocampal damage on haloperidol-induced catalepsy and apomorphineinduced stereotypic behaviors in the rat. Brain Res Dev Brain Res. 1993;75(2):213-22.

77. Chambers RA, Sentir AM, Conroy SK, Truitt WA, Shekhar A. Cortical-striatal integration of cocaine history and prefrontal dysfunction in animal modeling of dual diagnosis. Biol Psychiatry. 2010;67:788-92.

78. Chambers RA, McClintick JN, Sentir AM, Berg SA, Runyan M, Choi $\mathrm{KH}$, et al. Cortical-striatal gene expression in neonatal hippocampal lesion (NVHL)-amplified cocaine sensitization. Genes Brain Behav. 2013;12:564-75.

79. Berg SA, Sentir AM, Cooley BS, Engleman EA, Chambers RA. Nicotine is more addictive, not more cognitively therapeutic in a neurodevelopmental model of schizophrenia produced by neonatal ventral hippocampal lesions. Addict Biol. 2013

80. Berg SA, Sentir AM, Bell RL, Engleman EA, Chambers RA. Nicotine effects in adolescence and adulthood on cognition and alpha(4)beta(2)-nicotinic receptors in the neonatal ventral hippocampal lesion rat model of schizophrenia. Psychopharmacology (Berlin). 2015;232:1681-92.

81. Conroy SK, Rodd Z, Chambers RA. Ethanol sensitization in a neurodevelopmental lesion model of schizophrenia in rats. Pharmacol Biochem Behav. 2007;86:386-94.

82. Berg SA, Czachowski CL, Chambers RA. Alcohol seeking and consumption in the NVHL neurodevelopmental rat model of schizophrenia. Behav Brain Res. 2011;218:346-9.

83. Jeanblanc J, Balguerie K, Coune F, Legastelois R, Jeanblanc V, Naassila M. Light alcohol intake during adolescence induces alcohol addiction in a neurodevelopmental model of schizophrenia. Addict Biol. 2015;20:490-9.

84. Yang XH, Huang J, Lan Y, Zhu CY, Liu XQ, Wang YF, et al. Diminished caudate and superior temporal gyrus responses to effort-based decision making in patients with first-episode major depressive disorder. Prog Neuropsychopharmacol Biol Psychiatry. 2015;64:52-9.

85. Zhang L, Wang K, Zhu C, Yu F, Chen X. Trait Anxiety Has Effect on Decision Making under Ambiguity but Not Decision Making under Risk. PLoS One. 2015;10:e0127189.

86. Reckless GE, Andreassen OA, Server A, Ostefjells T, Jensen J. Negative symptoms in schizophrenia are associated with aberrant striato-cortical connectivity in a rewarded perceptual decisionmaking task. Neuroimage Clin. 2015;8:290-7.

87. Brown EC, Hack SM, Gold JM, Carpenter Jr WT, Fischer BA, Prentice KP, et al. Integrating frequency and magnitude information in decision-making in schizophrenia: An account of patient performance on the Iowa Gambling Task. J Psychiatr Res. 2015;66-67: $16-23$.

88. Lee D. Decision making: from neuroscience to psychiatry. Neuron. 2013;78:233-48.

89. Nasrallah NA, Clark JJ, Collins AL, Akers CA, Phillips PE, Bernstein IL. Risk preference following adolescent alcohol use is associated with corrupted encoding of costs but not rewards by mesolimbic dopamine. Proc Natl Acad Sci U S A. 2011;108: 5466-71.

90. Clark JJ, Nasrallah NA, Hart AS, Collins AL, Bernstein IL, Phillips PE. Altered risk-based decision making following adolescent alcohol use results from an imbalance in reinforcement learning in rats. PLoS One. 2012;7:e37357.

91. Boutros N, Semenova S, Liu W, Crews FT, Markou A. Adolescent intermittent ethanol exposure is associated with increased risky choice and decreased dopaminergic and cholinergic neuron markers in adult rats. Int J Neuropsychopharmacol. 2015;18. Authors demonstrated long lasting effects of binge-like alcohol exposure on both cholinergic and dopaminergic synthesis which is associated with increased risky choice when the reward is unlikely to be delivered. These data could explain the pursuit of risky behavior at adulthood (such as drug taking) in subject exposed to alcohol during adolescence. 